Communication

\title{
Telomere-related genomic instability in papillary thyroid cancers: a preliminary study
}

\author{
AlineRangel-Pozzo ${ }^{1, \sharp}$, TinucciaDettori ${ }^{2, \sharp}$, Daniela Virginia Frau ${ }^{2}$, John Gartner ${ }^{3}$,Garbor Fisher ${ }^{3}$, Roberta \\ Vanni' ${ }^{2}$, Sabine Mai $^{1}$ and Paola Caria ${ }^{2, *}$ \\ 1 Cell Biology, Research Institute of Oncology and Hematology, CancerCare Manitoba, University of \\ Manitoba, Winnipeg, MB R3E 0V9, Canada; aline.rangelpozzo@umanitoba.ca; sabine.mai@umanitoba.ca \\ 2 Department of Biomedical Sciences, University of Cagliari, 09042 Monserrato, Italy; paola.caria@unica.it \\ 3 Department of Pathology and Immunology, Max Rady College of Medicine, Rady Faculty of Health \\ Sciences, University of Manitoba, Winnipeg, MB R3E 0W2, Canada; John.Gartner@umanitoba.ca;
}

Gabor.Fischer@umanitoba.ca

\author{
*Correspondence: paola.caria@unica.it; Tel.: (+39 0706754103) \\ \#These authors contributed equally to this work
}

\begin{abstract}
Papillary thyroid carcinoma (PTC) has two main histologic variants: classical-PTC (CL-PTC) and follicular variant PTC (FV-PTC). Recently, due to its similar features to benign lesions, the encapsulated FV-PTC variant was reclassified as noninvasive follicular thyroid neoplasm with papillary-like nuclear features (NIFTP). Nonetheless, specific molecular signatures are not yet available. It is well known that telomere-related genome instability is caused by inappropriate DNA repair of dysfunctional telomeres and that mechanisms involved in the damaged telomere repair processing may led to detrimental outcomes, altering the 3D nuclear telomere and genome organization in cancer cells. This pilot study aimed to evaluate whether a specific nuclear telomere architecture might characterize NIFTP, potentially distinguishing it from other PTC histologic variants. Our findings demonstrate that 3D telomere profiles of CL-PTC and FV-PTC were different from NIFTP and that NIFTP more closely resembles follicular thyroid adenoma (FTA). NIFTP has longer telomeres than CL-PTC and FV-PTC samples and telomere length overlaps in NIFTP and FTA. There was no association between BRAF expression and telomere length in all tested samples. Our data showing that 3D nuclear telomere organization is altered differently in thyroid cancer variants, suggest that this parameter might guide clinical management of NIFTP. Although further investigations in a larger cohort of patients are necessary to corroborate our observations, telomere-related genomic instability might be of value in the diagnosis of NIFTP and allow for a more appropriate selection of the correct treatment.
\end{abstract}

Keywords: Papillary thyroid cancer, noninvasive follicular thyroid neoplasm with papillary-like nuclear features, follicular adenoma, telomere-related genomic instability 


\section{Introduction}

Thyroid cancer is one of the most common malignant endocrine neoplasms, and papillary thyroid carcinoma (PTC) constitutes approximately $80 \%$ of all thyroid cancer cases [1]. PTC consists of two main histological variants: classical-PTC (CL-PTC) and follicular variant PTC (FV-PTC). FV-PTC is divided into two sub-groups based on two different morphological aspects: non-encapsulated and encapsulated nodules [2]. Recently, the encapsulated variant was reclassified as noninvasive follicular thyroid neoplasm with papillary-like nuclear features (NIFTP), because it shows features similar to non-malignant lesions [3]. The Cancer Genome Atlas (TCGA) study [4] has demonstrated a strong correlation between genetic alterations and histologic phenotypes, resulting in the identification of two distinct molecular subgroups: the BRAFV600E-like nodules, which show the true papillary architecture, and RAS-like nodules, with a follicular-pattern that includes follicular adenoma, follicular carcinoma, and FV-PTC [5] .Although no specific molecular signatures have been identified, the typical molecular profile of NIFTP is of RAS-like mutations [6]. Moreover, prevalence of TERT promoter mutations, mutations in the gene promoter of human telomerase reverse transcriptase, have been observed in advanced forms of PTC with a high level of recurrence and disease-specific mortality [7], and are not found in NIFTP.

It is well known that telomeres have an important role in preserving chromosomal stability and integrity. Undamaged telomeres prevent end-to-end fusions, degradation of the chromosome ends, and contribute to the adequate chromosome positioning within the nucleus [8]. Studies using quantitative 3D telomere imaging and quantitative analysis have shown that in contrast to normal cells, telomeres are organized in a distinct pattern within the 3D space of the normal cell nucleus, in contrast to cancer cells [9-11]. Recently, we highlighted the importance of telomere-related genomic instability in the tumorigenesis of PTC. By selecting PTC-derived cell lines characteristic of PTC tumors $B R A F^{V 600 E}-$ like subgroup, we demonstrated that cancer stem-like cells subpopulations (representing an early cancer-promoting subpopulation) had a trend toward a lower telomere shortening compared to the corresponding parental cells, representing the tumor bulk cells [12].The present pilot study was designed to explore the potential use of telomere "signatures" in the differentiation of the different PTC histotypes. In particular, considering that it is still not clear whether the biological features of NIFTP are similar to FTA or to FV-PTC, our findings suggest that the specific 3D pattern observed in NIFTP may provide an additional parameter in the differential diagnosis of indolent thyroid nodules and there by prevent unnecessary patient treatment.

\section{Results and Discussion}

\subsection{NIFTP has a 3D profile similar to FTA and different from classical and follicular variants of PTC}

To date, there is no specific molecular signature of NIFTP, since the available panels of mutations used to improve diagnostic accuracy of PTC are not optimal to differentiate NIFTP from CL-PTC and FV-PTC. In this context, we investigated the 3D telomere profiles of different PTC histotypes and FTA in search of specific characteristics that would differentiate between them from NIFTP. In our pilot study, we used fifteen FFPE thyroid neoplasms comprising CL-PTC $(n=4)$, FV-PTC $(n=3), \operatorname{NIFTP}(n=3)$, and FTA $(n=4)$. We compared their respective telomere parameters across and found a pattern differentiating FTA and NIFTP from FV-PTC and CL-PTC. FTA and 
NIFTP presented higher $(\uparrow)$ numbers of telomere signals, $\uparrow$ numbers of telomere aggregates, and $\uparrow$ total intensity when compared with FV-PTC and CL-PTC (Figure 1 and Figure 2). The fact that classical and follicular variant PTC displayed lower numbers of telomeres (Figure 1A) and shorter telomeres (Figure 1C) when compared with NIFTP and FTA, suggested that the decrease in the number of telomeres might be a consequence of telomere shortening. In this case, fewer telomeric (TTAGGG) repeats would be available for probe binding. These results corroborate findings in a previous study that demonstrated the association of short telomeres with classic and follicular variant of PTC and of long telomeres with benign thyroid nodules [13-14]. However, the dot plot for telomere aggregates in Figure 1B shows $\uparrow$ numbers of telomere aggregates for FTA and NIFTP in comparison with FV-PTC and CL-PTC cases. Telomere aggregates are cluster of telomeres, found in close proximity that cannot be further resolved as separate entities at an optical resolution limit of $200 \mathrm{~nm}$ [15;16]. Some of the aggregates represent telomere fusions that can generate dicentric chromosomes and breakage-fusion-bridge cycles, leading to chromosome rearrangements and ongoing chromosome instability, usually found in FTC and FTA. This chromosome instability, results in the aneuploidy and telomere association seen in classical cytogenetic studies [17]. Our results seem to follow this trend.
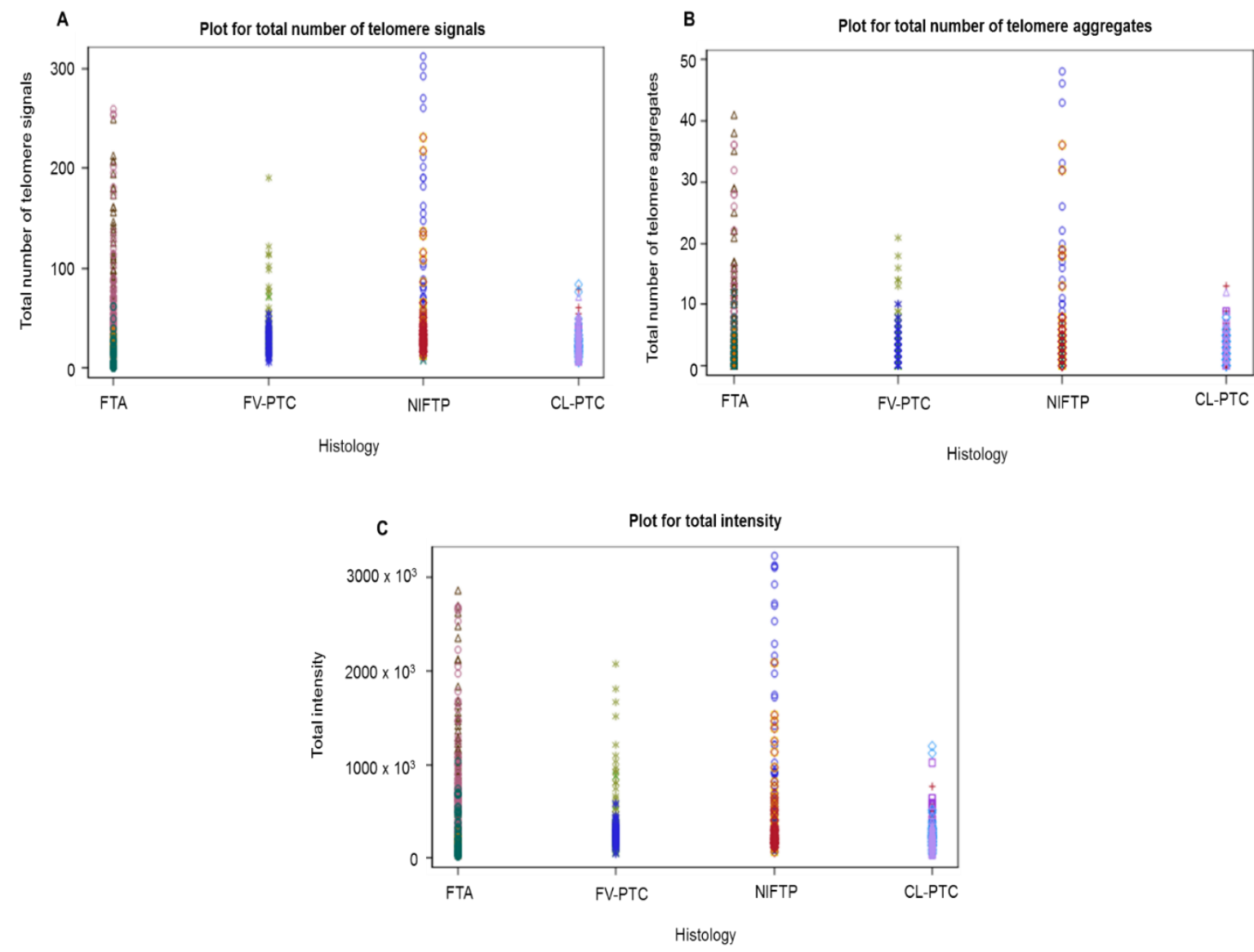

Figure 1: Dot plot of telomere parameters for FTA, FV-PTC, NIFTP, and CL-PTC cases. Each cell analyzed per histotype is represented in the graph. (A) Total number of telomere signals - a sum value representing the number of telomeres found in each cell population. (B) Total number of telomere aggregates - telomeres in proximity forming clusters that cannot be further resolved at an optical resolution limit of $200 \mathrm{~nm}$ - which, functionally, are fused telomeric signals or telomeres in 
close illegitimate proximity able to engage in recombination events. (C) Total telomere signal intensity (proportional to telomere length). The $x$-axis shows values for each parameter and the $\mathrm{y}$-axis refers to the cells analyzed in each histotype.

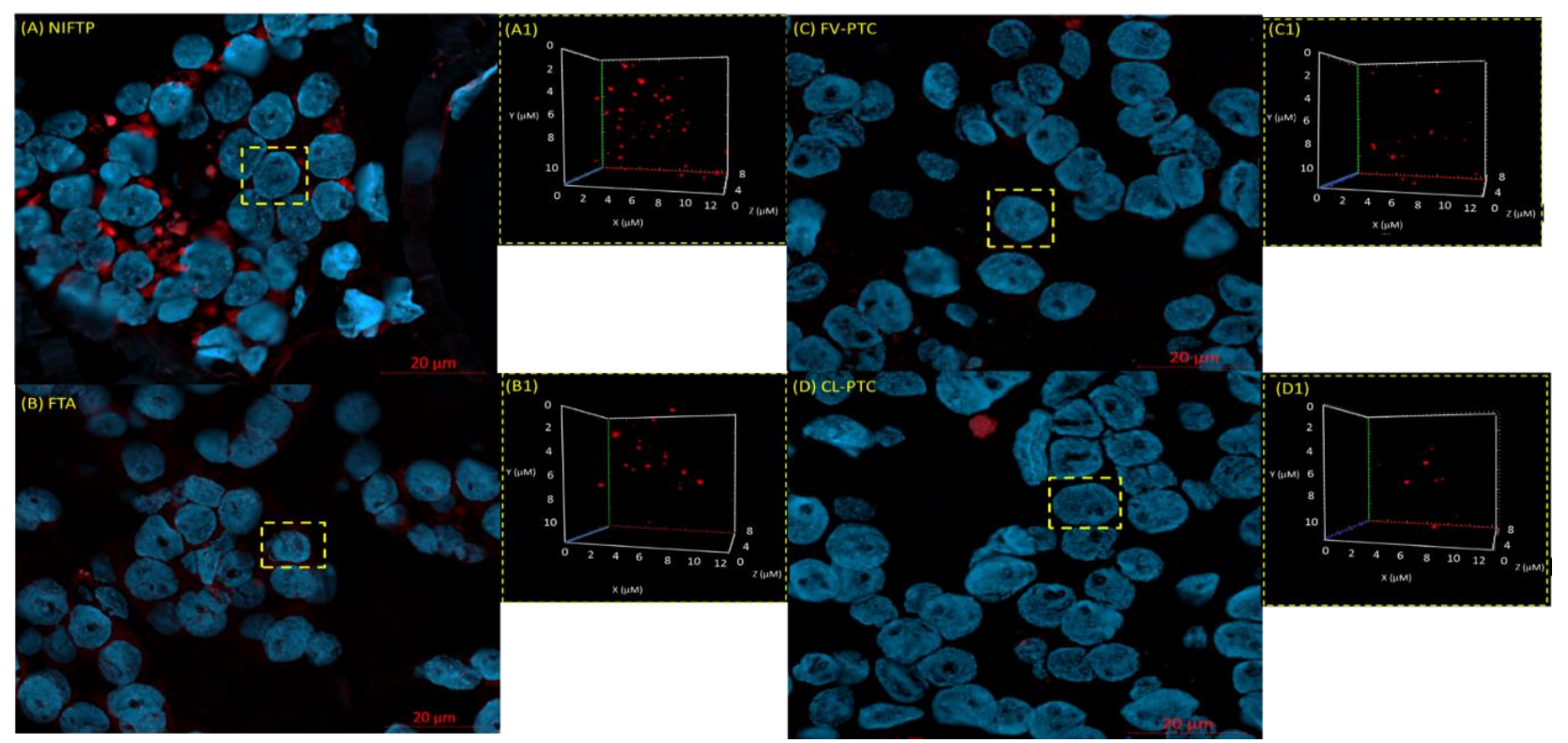

Figure 2: Differences in the 3D nuclear telomeric architecture between NIFTP (A), FTA (B), FV-PTC (C) and CL-PTC (D). Representative nuclei, counterstained with DAPI (blue) are shown for each histotype. Cy-3 labelled telomeres appear as red signals in the 3D figures in A1, B1, C1 and D1.

TheTeloView ${ }^{\circledR}$ analysis allowed us to divide the telomeric signals into four quartiles based on their intensity, creating distributions based on the frequency of signals with a specific intensity. We identified four cell subpopulations based on their telomeric signal intensity in arbitrary units (a.u.):cells with very short telomeres $(\leq 4000$ a.u.), cells with short telomeres (4001-7000 a.u), cell with medium telomeres (7001-13000 a.u), and cells with large telomeres(>13.000 a.u.). Interestingly, the frequency distribution of very short telomeres ( $\leq 4000 \mathrm{a} . \mathrm{u})$ showed a significant difference between the histotypes FTA/NIFTP and CL-PTC/FV-PTC (Figure 3). No significant differences were observed between FTA and NIFTP or between CL-PTC and PV-PTC. The figure 3 clearly shows that NIFTP parallels FTA telomere length, and that NIFTP has longer telomeres compared to classical and follicular variants of thyroid cancer. Our data clearly demonstrate that NIFTP has telomere signatures similar to FTA that is distinct from classical and follicular variants PTC. Although, NIFTP cannot be considered a benign lesion but rather a low-risk thyroid nodule, our results suggest and reinforce the idea that NIFTP are lesions more closely related to benign thyroid neoplasms, rather than to malignant lesions $[18,19]$. 


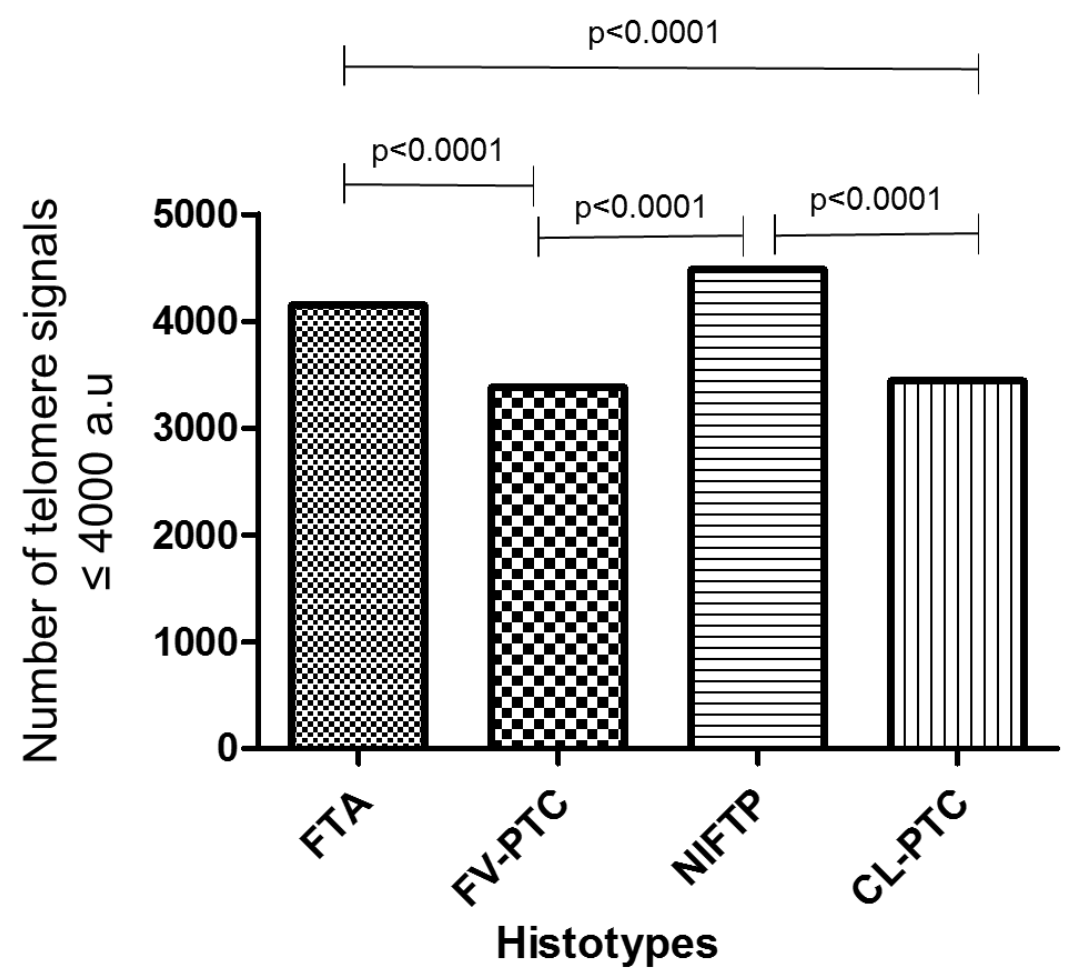

Figure 3: Bar plot of the differences in number of telomeres $\leq 4000$ a.u (arbitrary units) between CL-PTC, FV-PTC, FTA, and NIFTP. Cells with intensity of $\leq 4000$ a.u. represent very short telomeres. The $x$-axis assigns one box for each cell population analyzed (results of all analyzed cells). The y-axis refers to the number of telomeres $\leq 4000$ a.u. (showing only the significant $p$ values).

2.2 Telomere -related genomic instability is reflected in normal adjacent to tumor areas for CL-PTC and FV-PTC

Considering that telomeres length in healthy tissue can vary greatly from one patient to another, we also analyzed the 3D telomere profiles in normal adjacent to tumor areas (NAT). In general, as we expected, most of the telomere parameters were different between NAT and tumor areas for FTA and NIFTP. However, for CL-PTC and FV-PTC cases, we observed the opposite. Among the 3D telomeres parameters analyzed, we found that only the number of telomeres and total intensity in CL-PTC were significantly different between NAT and tumor areas, whereas in FV-PTC only the average intensity was different (Table 1 and Figure 4). The field effect theory has been invoked to explain this type of finding in different cancers. The theory suggests that molecular alterations develop in morphological normal-appearing epithelium tissue and contribute to the progression of carcinogenesis [20]. This seems to be the case for CL-PTC and FV-PTC. 
Table 1. 3D telomere parameters of thyroid tumor versus normal to adjacent tumor tissue CL-PTC-: classical-PTC; FV-PTC: follicular variant PTC; NIFTP: noninvasive follicular thyroid

\begin{tabular}{|c|c|c|c|c|}
\hline Histotype & $\begin{array}{c}\mathrm{n}^{\circ} \text { of telomere } \\
\text { signals }\end{array}$ & $\begin{array}{c}\mathrm{n}^{\circ} \text { of telomere } \\
\text { aggregates }\end{array}$ & $\begin{array}{l}\text { Average } \\
\text { intensity }\end{array}$ & $\begin{array}{c}\text { Total } \\
\text { intensity }\end{array}$ \\
\hline CL-PTC & $\begin{array}{c}\mathrm{p}<0.001 \\
(\downarrow N T A \times \mathrm{T} \uparrow)\end{array}$ & ns & ns & $\begin{array}{c}\mathrm{p}=0.0365 \\
(\downarrow N T A \times \mathrm{T} \uparrow)\end{array}$ \\
\hline FV-PTC & ns & ns & $\begin{array}{c}\mathrm{p}<0.001 \\
(\uparrow \mathrm{NTA} \times \mathrm{T} \downarrow)\end{array}$ & ns \\
\hline FTA & $\begin{array}{c}\mathrm{p}<0.001 \\
(\downarrow \mathrm{NTA} \times \mathrm{T} \uparrow)\end{array}$ & $\begin{array}{c}\mathrm{p}<0.001 \\
(\downarrow \mathrm{NTA} \times \mathrm{T} \uparrow)\end{array}$ & $\begin{array}{c}\mathrm{p}=0.0106 \\
(\downarrow \mathrm{NTA} \times \mathrm{T} \uparrow)\end{array}$ & $\begin{array}{c}\mathrm{p}<0.001 \\
(\downarrow \mathrm{NTA} \times \mathrm{T} \uparrow)\end{array}$ \\
\hline NIFTP & $\begin{array}{c}\mathrm{p}<0.001 \\
(\uparrow \mathrm{NTA} \times \mathrm{T} \downarrow)\end{array}$ & $\begin{array}{c}\mathrm{p}<0.001 \\
(\downarrow \mathrm{NTA} \times \mathrm{T} \uparrow)\end{array}$ & ns & $\begin{array}{c}\mathrm{p}<0.001 \\
(\uparrow \mathrm{NTA} \times \mathrm{T} \downarrow)\end{array}$ \\
\hline
\end{tabular}




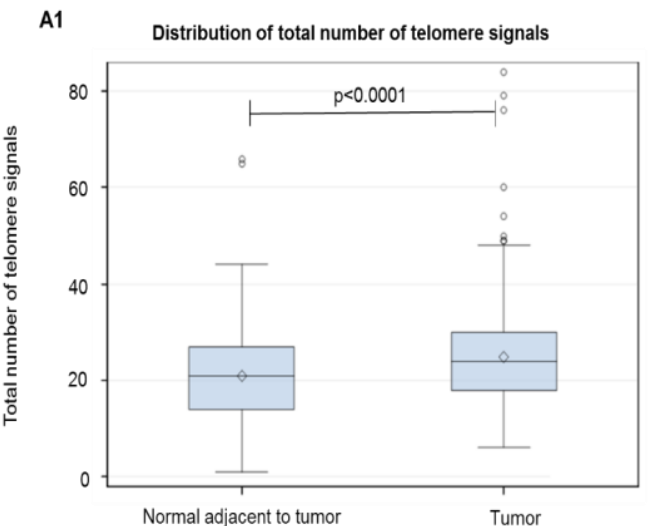

Classic-PTC (CL-PTC)

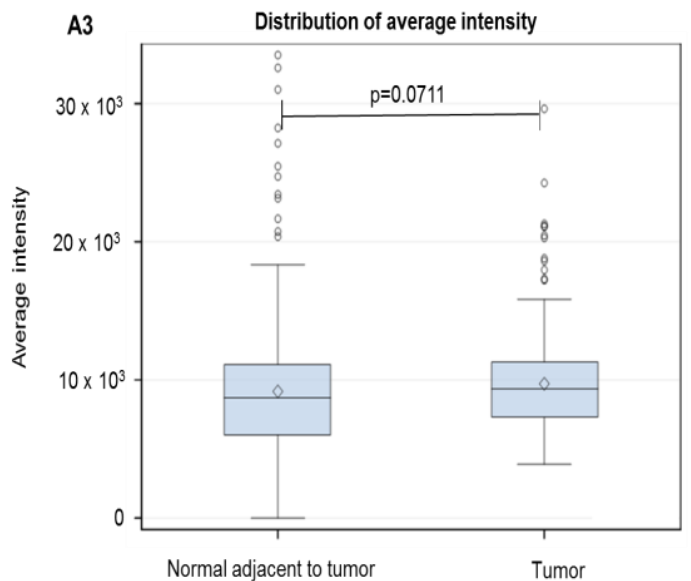

Classic-PTC (CL-PTC)

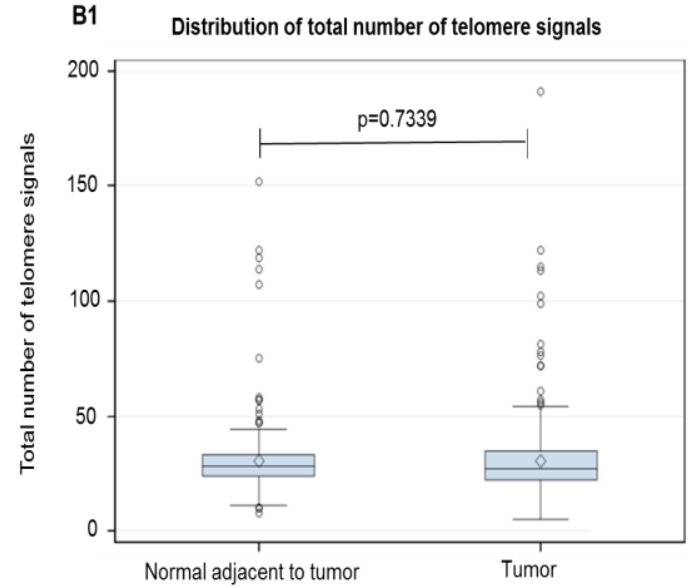

Follicular variant PTC (FV-PTC)

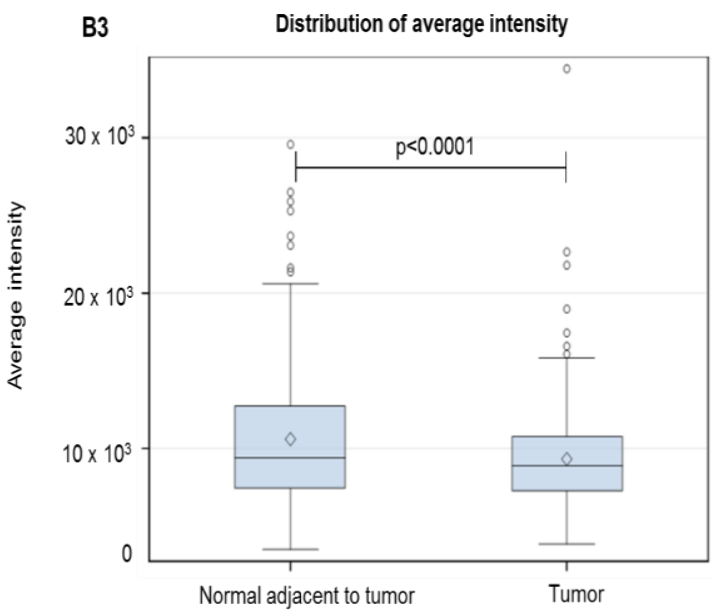

Follicular variant PTC (FV-PTC

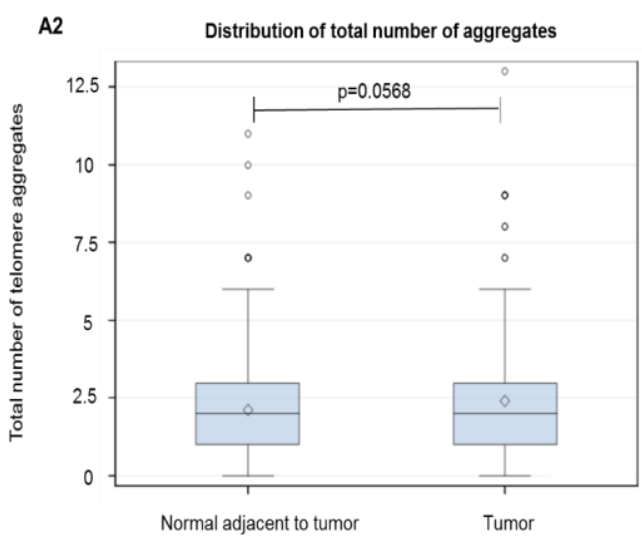

Classic-PTC (CL-PTC)

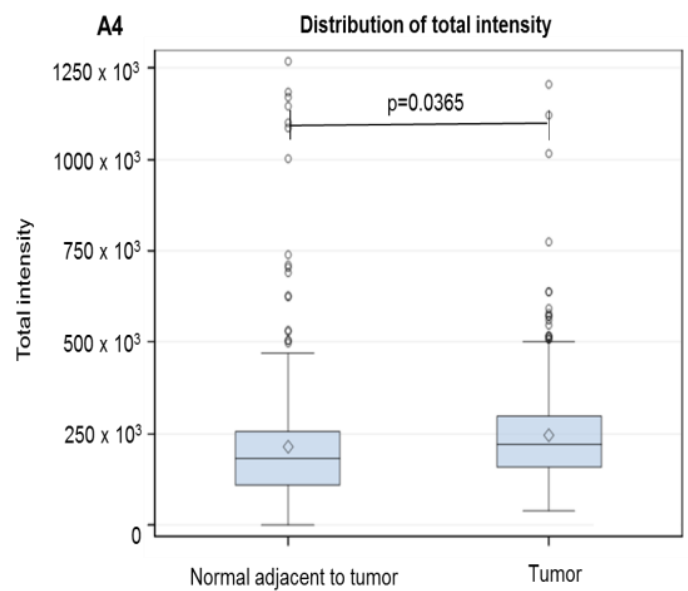

Classic-PTC (CL-PTC)

B2 Distribution of total number of aggregates

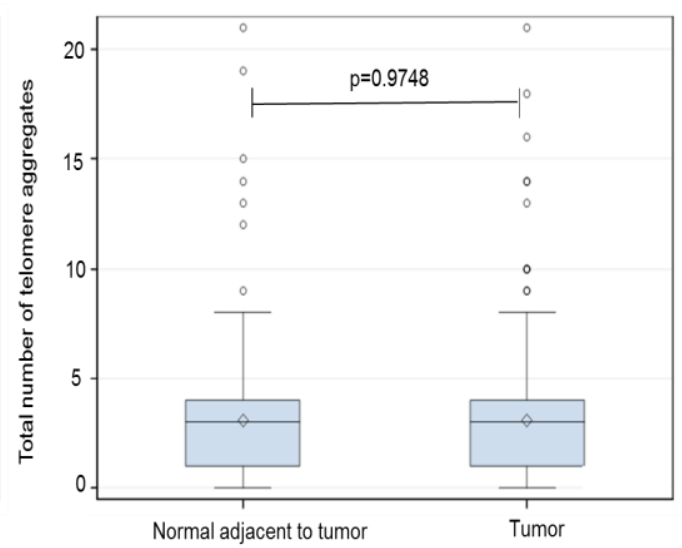

Follicularvariant PTC (FV-PTC)

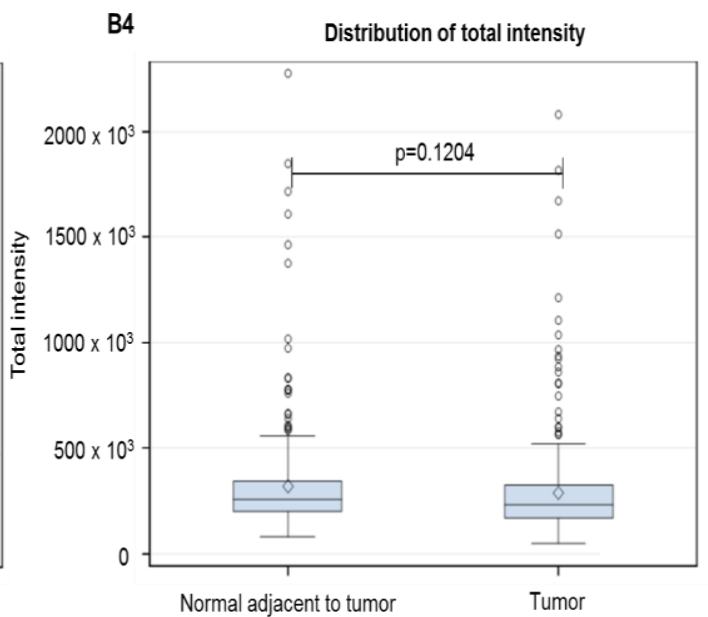

Follicularvariant PTC (FV-PTC) 

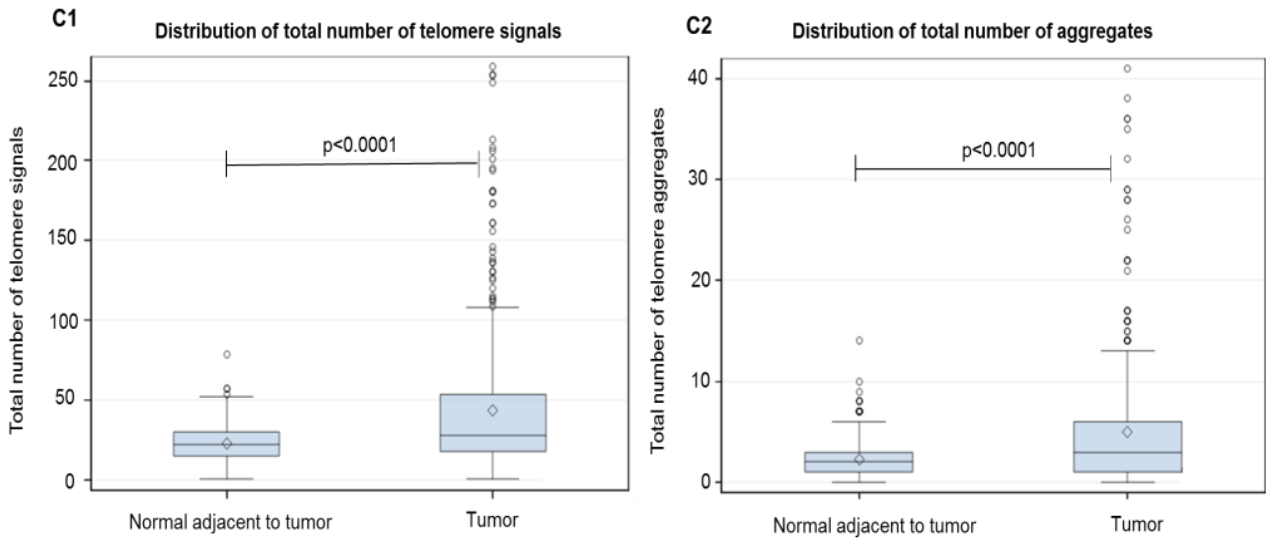

Follicular adenoma (FTA)

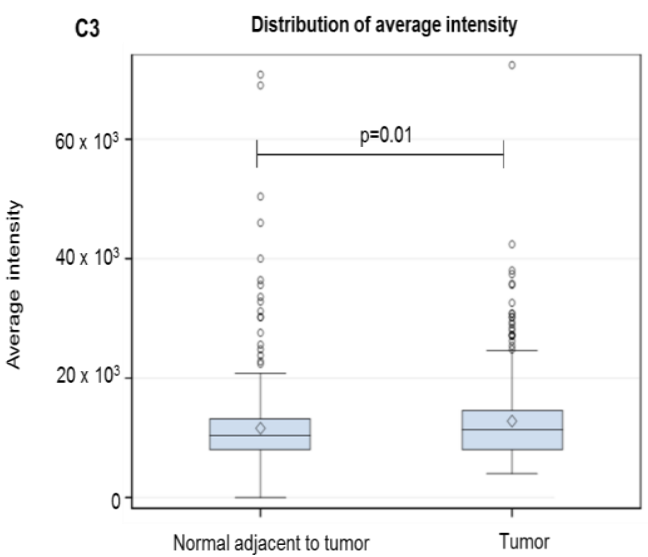

Follicular adenoma (FTA)
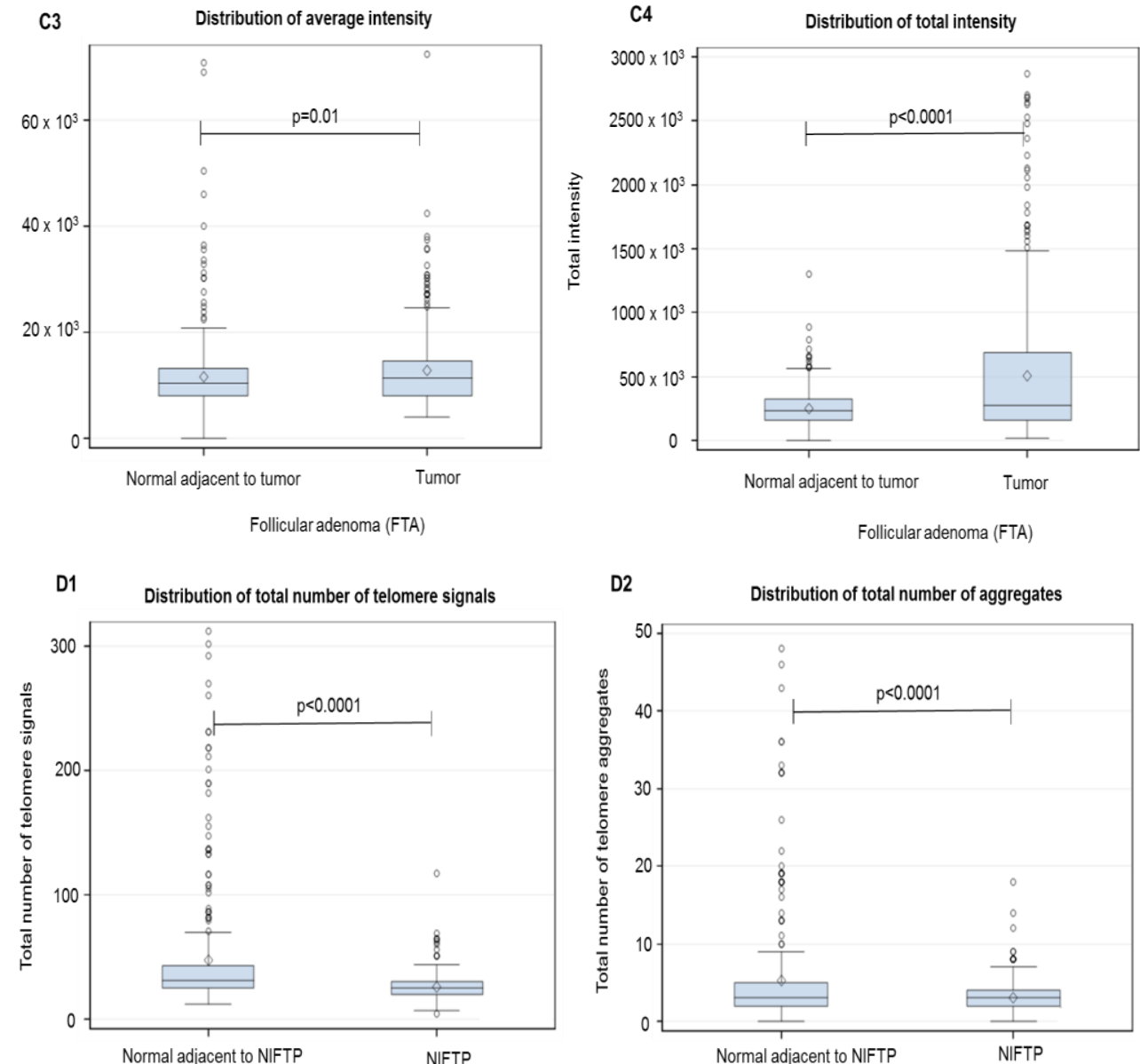

Follicular adenoma (FTA)
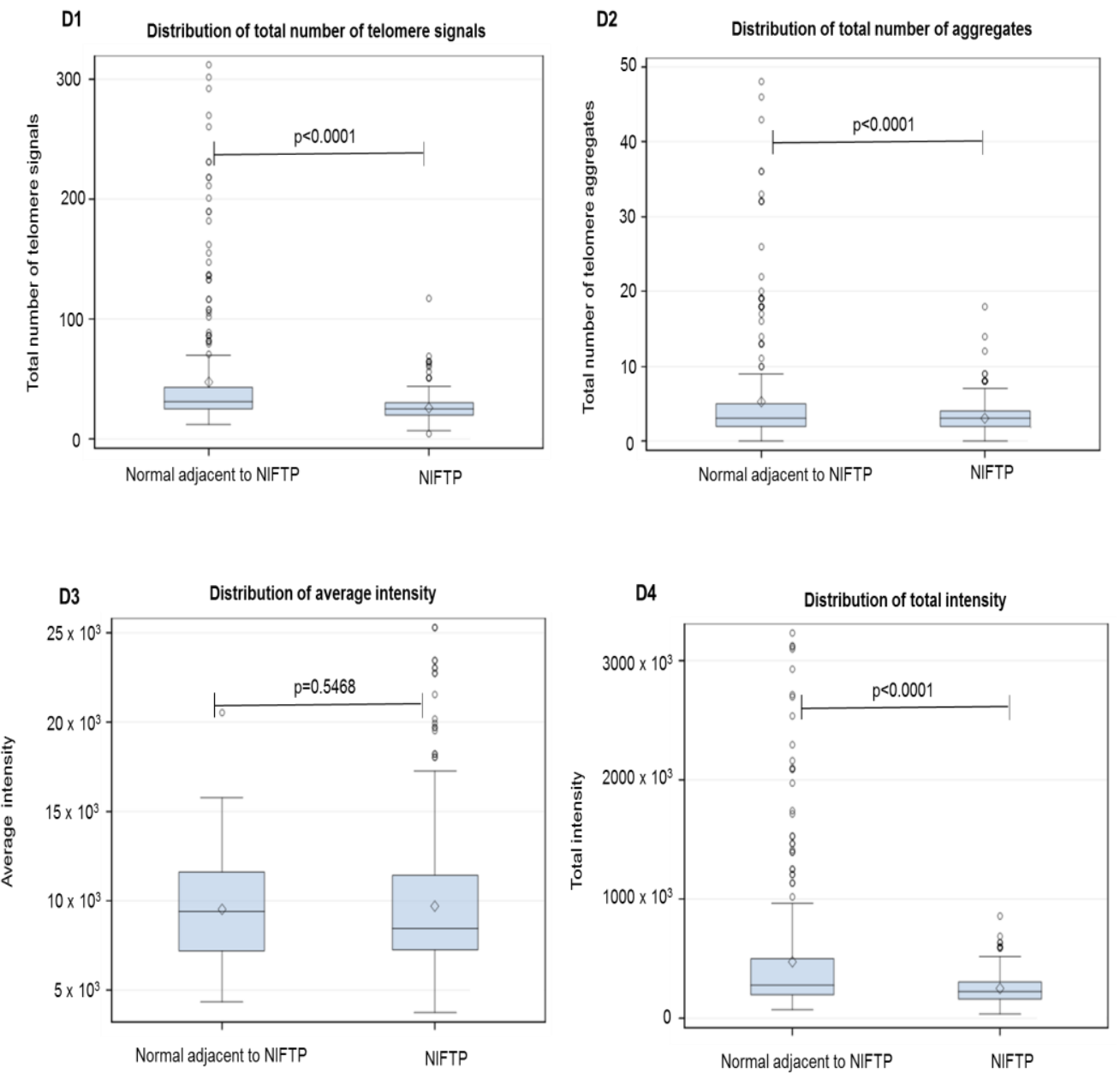
Figure 4: Bar plot of the differences in telomere parameters between CL-PTC (A), FV-PTC (B), FTA (C) and NIFTP (D) tumor and normal adjacent to tumor areas. (1) Total number of telomere signals a sum value representing the number of telomeres found in each cell population. (2) Total number of telomere aggregates - telomeres in close proximity forming clusters that cannot be further resolved at an optical resolution limit of $200 \mathrm{~nm}$. Functionally, telomeric aggregates functionally are fused telomeric signals or telomeres in close illegitimate proximity able to engage in recombination events. (3) Average intensity. (4) Total telomere signal intensity. For total intensity and average intensity, the position of each telomere is identified by using a threshold. Then, the center of gravity and the integrated intensity of each telomere is calculated as arbitrary units based on the number of sequences CCCTAA/probe intensity. The integrated intensity of each telomere is the appropriate parameter for determining the length of the telomere. The $x$-axis assigns one box for each cell population analyzed (results of all analyzed cells). The y-axis refers to parameters numbers, lower value, lower quartile, average (mean), median, and upper quartile.

\subsection{BRAFV600E expression was only observed in CL-PTC and FV-PTC cases}

Previous studies have demonstrated the importance of the BRAF mutation and RET/PTC rearrangements in promoting thyroid tumorigenesis [21;22]. We studied the presence of BRAF protein expression, indicative of $B R A F^{V 600 E}$ mutation, and RET rearrangements by FISH. First, we tested the antibody VE1 in PTC positive and negative for BRAFV600 by sequencing, and in normal thyroid tissues. The staining intensity, corresponding to the BRAF protein expression, was considered positive if unequivocal diffuse cytoplasmic staining of tumor cells was identified. We found that all BRAF positive samples presented BRAF protein expression, with a mean fluorescence intensity of 100 a.u (100 $\pm 10 \mathrm{SD})$, ranging from 81.5 to 113 . For BRAF negative cases, the mean fluorescence intensity was 19 a.u (19 \pm 0.7 SD), ranging from 19.8 to 20.7. Similar values were found in normal thyroid tissue ( $18 \pm 0.5 \mathrm{SD})$, as seen in Figure 5A, 5B, and 5C. Based on these observations, we analyzed BRAF expression /intensity. We found that 3 out of 15 cases were positive for BRAF expression, with fluorescence intensity between 81 to 86.5 (Supplementary table 1). All positive cases showed an unequivocal diffuse cytoplasmic staining with the BRAFV600E antibody in the majority of tumor cells (Supplementary Figure 2D and 2E) and they were classified as CL-PTC (2) and FV-PTC (1). On the molecular level, classic PTC is characterized by a high frequency of BRAF mutations (about $45 \%$ ), whereas in non-invasive encapsulated FV-PTC the presence of this mutation is rare [23;24]. Despite the small number of cases, our results are in line with these observations. The other CL-PTC (4) cases showed very heterogeneous staining and variable fluorescence intensity with an average of 47 a.u (Supplementary Figure 2F). The lack of specific criteria for the evaluation of BRAFV600E expression in thyroid tumors, by immunofluorescence, and the inability to perform sequencing due to low amount of DNA, made it difficult to interpret this last result. More cases are required in order to validate our observations. Moreover, all cases were negative for RET 
rearrangements, which could be related to the limited number of PTC cases in our study, considering the low frequency (10\% to $30 \%$ ) of this molecular alteration in adult PTC [25].
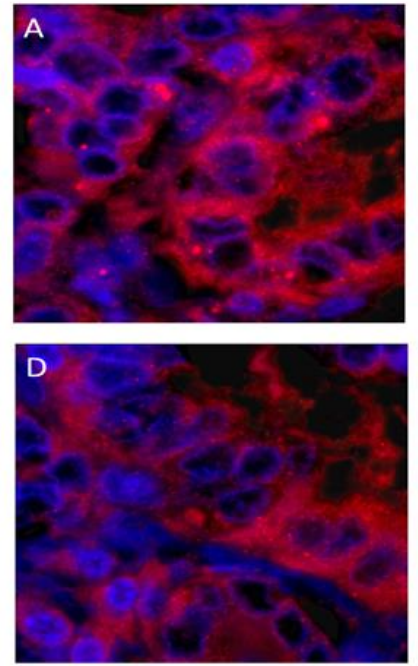
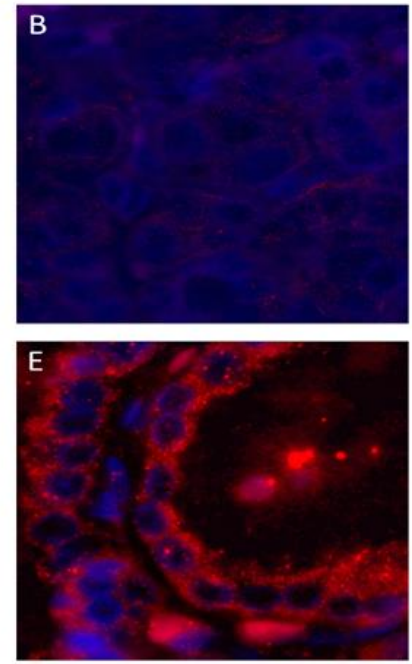
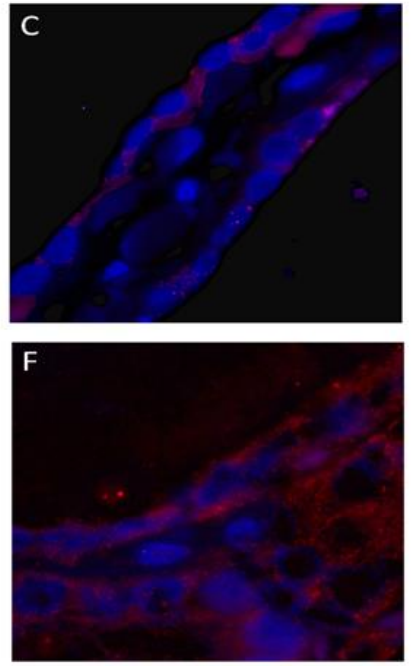

Figure 5: Representative images of BRAF V600E (VE1) immunofluorescence staining. BRAFV600E mutant PTC case (A), BRAFV600E - wild type PTC (B), normal thyroid tissue (C), CL-PTC and FV-PTC with an unequivocal diffuse cytoplasmic staining with the VE1 antibody in the majority of tumor cells (D and E), CL-PTC case with a heterogeneous staining and variable fluorescent intensity.

\section{Conclusions}

Our data indicate that $3 \mathrm{D}$ telomere profiles appear to be a promising tool to distinguish CL-PTC and FV-PTC from NIFTP. We demonstrated that NIFTP has a similar 3D telomere organization to FTA and is clearly different from classical and follicular variant PTC. These preliminary findings are in line with the view that NIFTP are lesions closer to non-malignant thyroid nodules and confirmed that short telomeres are a feature of PTC. This is the first study of 3D nuclear telomere organization in PTC. It provides a strong rationale for additional studies in a larger cohort of thyroid lesions. Moreover, if these data are confirmed, they may have diagnostic utility in facilitating the exact diagnosis of NIFTP, helping to predict the outcome of this tumor, and enabling patient-specific clinical management.

\section{Materials and Methods}

\subsection{Patient Samples}

This study received approval by the Research Ethics Board on human studies in Manitoba/Canada (HS21723; H2018:156). Informed consent was obtained from all the patients in accordance with the declaration of Helsinki. A total of 15 primary thyroid tumor tissue samples were obtained from the Health Sciences Centre (Winnipeg, Manitoba, Canada). The tumor and normal adjacent tissue (NAT) of formalin-fixed paraffin-embedded (FFPE)sections $(5 \mu \mathrm{m})$, were circled with a pen, after review of the corresponding hematoxylin and eosin stained sections by a pathologist (J.G and G.F). The experiments were blinded to the tumor characteristics and outcome data. The patient cohort 
included in this study was composed of $3(20 \%)$ men and 12 (80\%) women ages 31 to 67, with a median age of 51 years. Surgical specimens were classified according to the World Health Organization classification (WHO) [26]. Clinical, pathological, and molecular characteristics of the 15 patients are shown in Table 2.

Table 2. Clinical, pathological and molecular characteristics of patients

\begin{tabular}{|c|c|c|c|c|c|c|c|}
\hline $\begin{array}{c}\text { Case } \\
\mathrm{N}^{\circ}\end{array}$ & Sex & Age & Histotype & $\begin{array}{c}\text { TNM } \\
\text { Classification }\end{array}$ & $\begin{array}{c}\text { Tumor Size } \\
\text { (cm) }\end{array}$ & $\begin{array}{l}\mathrm{BRAF}^{\mathrm{V} 600 \mathrm{E}} \\
\text { expression }\end{array}$ & $\begin{array}{c}R E T / P T C \\
\text { rearrangments }\end{array}$ \\
\hline 1 & F & 57 & CL-PTC & pT2 & 3 & neg & neg \\
\hline 2 & F & 51 & CL-PTC & pT2N1Mx & 3.3 & neg & neg \\
\hline 3 & $\mathrm{~F}$ & 57 & CL-PTC & pT1 & 1.2 & pos & neg \\
\hline 4 & $\mathrm{~F}$ & 47 & CL-PTC & pT1aNX & 1 & pos & neg \\
\hline 5 & F & 67 & FV-PTC & pT3N1a & 1.7 & neg & neg \\
\hline 6 & M & 62 & FV-PTC & pT1bNxMx & 1 & neg & neg \\
\hline 7 & $\mathrm{~F}$ & 44 & FV-PTC & pT2MxNX & 4.2 & pos & neg \\
\hline 8 & M & 40 & NIFTP & na & 2.2 & neg & neg \\
\hline 9 & F & 66 & NIFTP & na & 1.5 & neg & neg \\
\hline 10 & $\mathrm{~F}$ & 31 & NIFTP & na & 1.3 & neg & neg \\
\hline 11 & M & 39 & NIFTP & na & 3.5 & neg & neg \\
\hline 12 & $\mathrm{~F}$ & 40 & FTA & na & 3.6 & neg & neg \\
\hline 13 & $\mathrm{~F}$ & 54 & FTA & na & 5 & neg & neg \\
\hline 14 & F & 62 & FTA & na & 1.6 & neg & neg \\
\hline 15 & $\mathrm{~F}$ & 31 & FTA & na & 4.2 & neg & neg \\
\hline
\end{tabular}

F: female; FTA: follicular thyroid adenoma; FV-PTC: follicular variant of papillary thyroid carcinoma; na: not avaible; NIFTP: noninvasive follicular thyroid neoplasm with papillary-like nuclear features; neg: negative; pos: positive; CL-PTC: classical papillary thyroid carcinoma.

\subsection{D quantitative fluorescence in situ hybridization (Q-FISH)}

Nuclei from 5- $\mu \mathrm{m}$ thyroid tissue sections underwent3D quantitative fluorescence in situ hybridization Q-FISH with Cy3-telomere probe-(TTAGGG)n (DAKO, Glostrup, Denmark). The hybridization procedure was performed as described previously with some modifications [27]. 
Briefly, paraffin-embedded tissue sections were deparaffinized with xylene 3 times for 15 min each and rehydrated in a series of ethanol $(100 \%, 70 \%$, and $50 \%)$ for 5 min each. Then the slides were incubated in $1 \mathrm{M} \mathrm{NaSCN}$ at $80^{\circ} \mathrm{C}$ for $20 \mathrm{~min}$ and washed in phosphate-buffered saline (PBS) twice for $5 \mathrm{~min}$ each, at room temperature (RT). Subsequently, the slides were incubated in $3.7 \%$ formaldehyde/1× PBS for $30 \mathrm{~min}$ and washed while shaking in 1× PBS 3 times for $5 \mathrm{~min}$ each, followed by $10 \mathrm{~min}$ of pepsin $(50 \mu \mathrm{g} / \mathrm{ml}) / 2 \mathrm{M} \mathrm{HCl}$ treatment at $37^{\circ} \mathrm{C}$. After hybridization with Peptide Nucleic Acid (PNA) telomeric probe (DAKO, Mississauga, ON, Canada), the slides were washed and counterstained with 4',6-diamino-2- phenylindole (DAPI; $0.1 \mu \mathrm{g} / \mathrm{mL}$ ) for $3 \mathrm{~min}$.

The 3D image analysis was carried out on 100 nuclei per tumor sample using AxioImager Z2 microscope (Carl Zeiss Canada Ltd). A 63x/1.4 oil objective lens (Carl Zeiss Canada Ltd.) was used for image acquisition. Eighty z-stacks were acquired at a sampling distance of $x, y: 102 \mathrm{~nm}$ and $z: 200$ $\mathrm{nm}$ for each slice to create optical sections. The acquisition time of 300 milliseconds for Cy3 (telomeres) was used, while the exposure times for the DAPI (nuclei) varied for each sample. The constrained iterative algorithm was used for deconvolution [28]. Deconvolved images were analyzed using the TeloView ${ }^{\circledR}$ v1.03 software program (Telo Genomics Corp., Toronto, ON, Canada) [15]. TeloView ${ }^{\circledR}$ measures in each cell six different telomeric parameters - telomeric signal intensity (telomere length), number of telomeric signals, number of telomere aggregates, nuclear volume, $a / c$ ratio, and distribution of telomeres relative to the nuclear periphery - generating specific 3D telomere profiles for each examined thyroid sample [12,29].

\subsection{BRAF expression and RET/PTC rearrangements}

BRAFV60E protein expression was detected using the anti-BRAFV600E (clone VE1) mutation-specific mouse monoclonal antibody (Abcam ab228461 Milano, Italy) in accordance with the protocol utilized in previous studies [30]. Images were obtained with an epifluorescence microscope (Olympus BX41) and charge-coupled device camera (Cohu), interfaced with the CytoVysion system (software 2.81 Applied Imaging, Pittsburg, PA, USA). In each section, tumor and normal area were evaluated, and ten randomly selected fields for each sample were acquired with a $100 \mathrm{X}$ objective. Sequencing-validated BRAFV600E mutation positive $(7$ samples) and negative (3 samples) PTC cases [31] were used as positive and negative controls, respectively. Normal thyroid tissue from the tumor-free contralateral lobe of five PTCs were used as control [32]. The Image J software (US National Institutes of Health, USA) was used to determine the fluorescence intensity as previously described [33]. The presence of RET/PTC variants was tested by FISH with home-made probes, using dual-colour break-apart strategy, as previously described [32]. The presence of $R A S$ mutations was not investigated.

\subsection{Statistical Analysis}

To compare telomere parameters among samples with different histology nested factorial analysis of variance was used. We also compared telomeric features of cells from NAT and from tumor area for each patient with a different histology using the Wilcoxon rank sum tests. Significance levels were set at $\mathrm{p}=0.05$. 
Supplementary Materials: Supplementary Table 1. Clinical, pathological and molecular characteristics of patients. Supplementary Figure 1: Bar plot of the differences in telomere parameters between CL-PTC (A), FV-PTC (B), FTA (C), and NIFTP (D) tumor and normal adjacent to tumor areas. Supplementary Figure 2: Representative images of of BRAF V600E (VE1) immunofluorescence staining

Author Contributions: Experiments, T.D.; D.V.F.;P.C.; Analysis, P.C and A-R.P.; resources, J.G. and G.B.; writing-original draft preparation, P.C and A-R.P.; writing-review and editing, A.R-P.; J.G.; G.F.; R.V.; S.M.; P.C.; supervision, P.C. All authors have read and agreed to the published version of the manuscript.

Funding: This work was supported by the Research Integrative Fund (FIR) of the University of Cagliari to P.C, T.D and R.V.

Acknowledgments: The authors would like to thank Telo Genomics Corp. for the use of TeloView® software platform, Mary Cheang for the statistical analysis and Elizabete Cruz for helping in the manuscript preparation. The authors also thank the Genomic Centre for Cancer Research and Diagnosis (GCCRD) for imaging. The GCCRD is funded by the Canada Foundation for Innovation and supported by CancerCare Manitoba Foundation, the University of Manitoba and the Canada Research Chair Tier 1 (SM). The GCCRD is a member of the Canadian National Scientific Platforms (CNSP) and of Canada BioImaging. The authors thank Department of Pathology - Health Sciences Centre (Winnipeg, Manitoba, Canada) for supporting this project with thyroid tissue samples.

Conflicts of Interest: S.M. is a shareholder, director and chair of the clinical and scientific advisory board of Telo Genomics Corp. (Toronto ON, Canada). The other authors declare that they have no conflicts of interest.

\section{References}

1. Davies L.; Welch H.G. Increasing incidence of thyroid cancer in the United States, 1973-2002. JAMA 2006, 295, 2164-7.

2. Liu, J.; Singh, B.; Tallini, G.; et al. Follicular variant of papillary thyroid carcinoma: a clinicopathologic study of a problematic entity. Cancer 2006, 107, 1255-1644.

3. Nikiforov, Y.E.; Seethala, R.R.; Tallini, G.; et al. Nomenclature revision for encapsulated follicular variant of papillary thyroid carcinoma: A paradigm shift to reduce overtreatment of indolent tumors. JAMA Oncol. 2016, 2, 1023-9.

4. Cancer Genome Atlas Research Network. Integrated genomic characterization of papillary thyroid carcinoma. Cell, 2014, 159, 676-690.

5. Stransky, N.; Cerami, E.; Schalm, S.; Kim, J.L.; Lengauer, C. The landscape of kinase fusions in cancer. Nat commun. 2014, 5, 4846.

6. Alves, V.A.F.; Kakudo, K.; LiVolsi, V.; Lloyd, R.V.; Nikiforov, Y.E.; Nosé, V.; Papotti, M.; Thompson, L.D.R. Noninvasive Follicular Thyroid Neoplasm With Papillary-Like Nuclear Features (NIFTP): Achieving Better Agreement By Refining Diagnostic Criteria. Clinics, 2018; 73, e576.

7. Melo, M.; da Rocha, A.G.; Vinagre, J.; Sobrinho-Simões, M.; Soares, P. Coexistence of TERT promoter and BRAF mutations in papillary thyroid carcinoma: added value in patient prognosis?. J Clin Oncol. 2015, 33, 667-668. 
8. de Lange, T. How telomere solve the end-protection problem. Science, 2009, 326, 948-952.

9. Klewes, L.; Vallente, R.; Dupas, E.; et al. Three-dimensional Nuclear Telomere Organization in Multiple Myeloma. Transl Oncol. 2013; 6, 749-56.

10. Knecht, H.; Sawan, B.; Lichtensztejn, D.; et al. The 3D nuclear organization of telomeres marks the transition from Hodgkin to Reed-Sternberg cells. Leukemia, 2009, 23, 565-573.

11. Kuzyk, A.; Gartner, J.; Mai S. Identification of Neuroblastoma Subgroups Based on Three-Dimensional Telomere Organization. Transl Oncol. 2016, 9, 348-56.

12. Caria, P.; Dettori, T.; Frau, D.V.; et al. Characterizing the three-dimensional organization of telomeres in papillary thyroid carcinoma cells. J Cell Physiol. 2019, 234, 5175-5185.

13. Wang, Y.; Meeker, A.K.; Kowalski, J.; et al. Telomere length is related to alternative splice patterns of telomerase in thyroid tumors. Am J Pathol. 2011, 179, 1415-24.

14. Capezzone, M.; Cantara, S.; Marchisotta, S.; et al. Telomere length in neoplastic and nonneoplastic tissues of patients with familial and sporadic papillary thyroid cancer. J Clin Endocrinol Metab. 2011, 96, 1852-6.

15. Vermolen, B.J.; Garini, Y.; Mai, S.; et al. Characterizing the three-dimensional organization of telomeres. Cytometry A. 2005, 67, 144-150.

16. Caporali, A.; Wark, L.; Vermolen, B.J.; et al. Telomeric aggregates and end-to-end chromosomal fusions require myc box II. Oncogene, 2007, 26, 1398-1406.

17. Caria, P.; Vanni, R. Cytogenetic and molecular events in adenoma and well-differentiated thyroid follicular-cell neoplasia. Cancer Genet Cytogenet. 2010, 203, 21-9.

18. Amendoeira, I.; Maia, T.; Sobrinho-Simões, M. Non-invasive follicular thyroid neoplasm with papillary-like nuclear features (NIFTP): impact on the reclassification of thyroid nodules. Endocr Relat Cancer. 2018, 25, 247-258.

19. Zajkowska, K.; Kopczyński, J.; Góźdź, S.; Kowalska, A. Noninvasive follicular thyroid neoplasm with papillary-like nuclear features: a problematic entity. Endocr Connect. 2020, 9, 47-58.

20. Chai, H.; and Brown, R.E. Field Effect in Cancer-An Update. Annals of Clinical E Laboratory Science, 2009, $39,331-7$.

21. Song, Y.S.; Park, Y.J. Genomic Characterization of Differentiated Thyroid Carcinoma. Endocrinol Metab. 2019, 34, 1-10.

22. Ciampi, R.; Nikiforov Y.E. RET/PTC Rearrangements and BRAF Mutations in Thyroid Tumorigenesis. Endocrinology, 2007, 148, 936-941.

23. Smith, R.A.; Salajegheh, A.; Weinstein, S.; et al. Correlation between BRAF mutation and the clinicopathological parameters in papillary thyroid carcinoma with particular reference to follicular variant. Hum Pathol. 2011, 42, 500-506.

24. Rivera, M.; Ricarte-Filho, J.; Knauf, J.; Shaha, A.; et al. Molecular genotyping of papillary thyroid carcinoma follicular variant according to its histological subtypes (encapsulated vs infiltrative) reveals distinct BRAF and RAS mutation patterns. Mod Pathol. 2010, 23, 1191-1200.

25. Valentina, D.; Yakushina, L.V.; Lerner and Alexander, V. Lavrov. Gene Fusions in Thyroid Cancer. Thyroid, 2018, 28: 158-167.

26. De Lellis, R.; Lloyd, R.; Heitz, P.; et al. Pathology and genetics of tumours of endocrine organs. World Health Organization classification of tumours. IARC. 2004, 8, 183-6. 
27. Louis, S.F.; Vermolen, B.J.; Garini, Y.; et al. c-Myc induces chromosomal rearrangements through telomere and chromosome remodeling in the interphase nucleus. Proc. Natl. Acad. Sci. USA, 2005, 102, 9613-9618.

28. Schaefer, LH.; Schuster, D.; Herz, H. Generalized approach for accelerated maximum likelihood-based image restoration applied to three-dimensional fluorescence microscopy. J Microsc. 2001, 204, 99-107.

29. Rangel-Pozzo. A.; Corrêa de Souza, D.; Schmid-Braz, A.T.; et al. 3D Telomere Structure Analysis to Detect Genomic Instability and Cytogenetic Evolution Myelodysplastic Syndromes. Cells, 2019, 8, 304.

30. Contu, F.; Rangel-Pozzo, A.; Trokajlo, P.; et al. Distinct 3D Structural Patterns of Lamin A/C Expression in Hodgkin and Reed-Sternberg Cells. Cancers (Basel), 2018, 10, 286.

31. Caria, P.; Cantara, S.; Frau, D.V.; et al. Genetic Heterogeneity of HER2 Amplification and Telomere Shortening in Papillary Thyroid Carcinoma. Int J Mol Sci. 2016,17, 1759.

32. Caria, P.; Dettori, T.; Frau, D.V.; et al. Assessing RET/PTC in thyroid nodule fine-needle aspirates: the FISH point of view. Endocr Relat Cancer, 2013, 20, 527-36.

33. Costa, G.; Morelli, M.; \& Simola, N. Progression and Persistence of Neurotoxicity Induced by MDMA in Dopaminergic Regions of the Mouse Brain and Association with Noradrenergic, GABAergic, and Serotonergic Damage. Neurotoxicity Research, 2017, 32, 563-574. 